\title{
OPEN Accuracy of core needle biopsy for histologic diagnosis of soft tissue sarcoma
}

\author{
J. Kiefer ${ }^{1}$, M. Mutschler ${ }^{1}$, Ph. Kurz ${ }^{2}$, G. B. Stark ${ }^{1}$, H. Bannasch ${ }^{3}$ \& F. Simunovic ${ }^{1 \bowtie}$
}

The biopsy technique of choice in soft tissue sarcoma (STS) diagnosis is controversial. We examined the diagnostic accuracy of percutaneous core needle biopsy (CNB) and compared it to open incisional biopsy. A retrospective study included 91 incisional biopsies and 102 CNBs. A pair-match investigation was conducted on 19 patient pairs, comparing sensitivity, specificity, and diagnostic accuracy. Furthermore, we investigated the role of molecular pathology in sarcoma diagnostics. In 81/91 (89\%) patients with incisional biopsy, the entity was confirmed by definitive pathology, whereas this was the case in $89 / 102(87 \%)$ CNB patients $(p=0.52)$. Grading remained unchanged in $46 / 55(84 \%)$ of incisional and $54 / 62(87 \%)$ of CNBs $(p=0.61)$. The pair matched analysis showed that the correct entity was determined in $96 \%$ of incisional and $97.6 \%$ of core needle biopsies. The time between the initial consultation and the interdisciplinary tumor board's treatment recommendation was shorter in core needle biopsies ( 8.37 vs. 15.63 days; $p<0.002$ ). Incisional biopsies led to two wound infections and one hematoma, whereas wound infection occurred in one patient after CNB. CNB leads to faster diagnosis while reaching the same histological accuracy and is less burdensome for patients. Still, surgeons need to remain aware of the possibility of biopsy failure.

Soft tissue sarcomas are a rare and highly heterogeneous group of malignancies, with over 50 entities ${ }^{1,2}$. Classification of sarcoma is undergoing regular revision, and research into molecular mechanisms of development and progression of sarcoma subtypes continues to translate into pathological diagnostics ${ }^{3-5}$. Correct histological diagnosis and tumor grading are paramount, as they dictate the type and sequence of therapeutic modalities. Traditionally open incisional biopsy delivered the best quality tissue for an accurate diagnosis with the lowest rate of false biopsies. However, this procedure requires general anesthesia, and complications such as hematoma and wound infection occur in up to $16 \%$ of cases $^{6}$. Such complications reduce patients' well-being and have adverse effects on the overall treatment outcome, as they can cause tumor spread and delay neoadjuvant treatment.

Less-invasive soft tissue tumor biopsy methods, such as percutaneous core needle biopsy $(\mathrm{CNB})$ and fineneedle aspiration biopsy, have emerged as alternatives. These procedures are performed in the office under local anesthesia. Obvious benefits include faster diagnosis, a lower burden for the patient and the hospital resources, and a lower complication rate. Downsides of percutaneous methods are the inability to visually discern between tumor and normal surrounding tissue, leading to a higher rate of false-negative biopsies, difficulty in accounting for the heterogeneous architecture of specific tumors, technical issues within anatomically challenging locations, and a limited amount of tissue gained.

Accordingly, earlier reports have criticized the lower accuracy of CNB concerning tumor entity and grading $^{7-12}$. A 2009 meta-analysis on 35 studies has found that incisional biopsy has had the highest diagnostic accuracy, followed by CNB, which has been more accurate than fine-needle aspiration biopsy ${ }^{8}$. A single prospective work, which has compared all three biopsy techniques on every tumor, has confirmed the three methods' proposed hierarchy ${ }^{6}$. Newer studies, including a comprehensive 2020 meta-analysis, have reported comparable accuracy of percutaneous $\mathrm{CNB}$ and incisional biopsy, and an increasing number of centers have cited CNB as the method of the first choice in diagnosing soft tissue tumors ${ }^{13-23}$. Even though the literature is heterogeneous regarding the most exact biopsy procedure, a trend over the last decade seems to favor CNB. CNB has thus found recognition as the method of choice in the 2018 guidelines of the National Comprehensive Cancer Network (NCCN) for soft tissue tumor biopsy ${ }^{24}$.

${ }^{1}$ Department of Plastic and Hand Surgery, Freiburg University Medical Center, Freiburg, Germany. ${ }^{2}$ Department of Pathology, Freiburg University Medical Center, Freiburg, Germany. ${ }^{3}$ Department of Plastic, Hand and Aesthetic Surgery, Schwarzwald-Baar-Klinikum Villingen-Schwenningen, Villingen-Schwenningen, Germany. ${ }^{\square}$ email: filip.simunovic@uniklinik-freiburg.de 
This study aims to report our institutional experience and investigate the diagnostic accuracy, sensitivity, specificity, safety, and time to diagnosis of CNB in comparison to open incisional biopsy. Furthermore, we analyze the role of molecular pathology in sarcoma diagnostics in our institution.

\section{Patients and methods}

This study is based on a prospective and continuously updated database of patients with suspected soft tissue sarcoma referred to the Department of Plastic and Hand Surgery at the Freiburg University Medical Center between 2003 and December 2020. The institution is a tertiary referral center and an academic teaching hospital. We introduced the core needle biopsy in our clinic in July 2015. This study reviewed all patients with suspected malignancy, excluding patients with radiologically and clinically inconspicuous masses (e.g., lipoma). Data were compiled from medical records as well as pathology reports and collected anonymously. Patient demographics, tumor type, localization, tumor grading, modality of neoadjuvant and adjuvant therapy and anatomical distribution were collected. The average age at the time of the first operation was $58.95 \pm 16.3$ in the IB and $61.32 \pm 18.48$ in the CNB group $(p=0.47)$ years.

National Comprehensive Cancer Network (NCCN) and M stage, grading, anatomical distribution, as well as the type of neoadjuvant and adjuvant radio- and chemotherapy were recorded (Table 1).

In the first part of the study, we retrospectively reviewed all biopsies, recording the number of samples, the modality of molecular diagnostics performed, tumor entity, and tumor grading (Fig. 1). We then compared these findings to the definitive resection specimens to evaluate the accuracy of diagnosis regarding malignancy, tumor entity, and grading. In the second part of the study, we performed a matched-pair analysis according to the following criteria: gender, age, tumor entity, localization, size and depth of the tumor (epi-/subfascial), and grading (Fig. 1). We assigned 38 patients to 19 pairs, which we further analyzed regarding diagnostic accuracy and time from initial consultation to case presentation in the interdisciplinary tumor board, time from initial consultation to initiation of therapy, and time from biopsy to initiation of therapy. Complications, such as wound infection and hematoma, were noted. No patients were lost to follow up, as they all proceeded to receive treatment at the Freiburg University Medical Center. We further reviewed the diagnostic accuracy as a function of time, asking whether there was a change in diagnostic accuracy during the study period. Finally, we reviewed whether the use of molecular testing was associated with higher diagnostic accuracy and which gene alterations and mutations were most frequently found in our population.

A board-certified plastic surgeon specializing in sarcoma surgery performed all biopsies, tumor resection, and reconstruction. The CNBs were performed in an outpatient setting, usually during the initial consultation. After obtaining informed consent, local anesthesia was administered, and the site of the biopsy was prepared. The surgeon then carefully examined the preoperative MRI scans for localization and heterogeneity of the soft tissue tumor to determine which section of the mass was most representative for biopsy. The skin was incised with an $11^{\circ}$ blade and the biopsy was performed using the 12 Gauge BIP-HistoCore HC needle (Biomed. Instrumente \& Produkte $\mathrm{GmbH}$, Türkenheim, Germany). If possible, the mass was palpated by the non-dominant hand during the biopsy (Fig. 2). In some cases, sonographic guidance was used to optimize the accuracy of the CNB. At least three tissue probes (mean 3.59 \pm 2 ; min. 1 and max. 12) were taken and examined visually, as the tumor tissue could often be differentiated macroscopically from fatty or muscle tissue. Tissue cylinders were then sent for pathologic analysis in formaldehyde.

Incisional biopsies were performed under general anesthesia on an in-patient basis. Following the skin incision, the soft tissue compartments were dissected until the tumor was identified, and a representative section of the tumor mass was biopsied under visual control through a wedge resection. Following pathology workup and staging, the case was presented at the interdisciplinary sarcoma board of the Freiburg University Medical Center, where the type and sequence of therapeutic modalities were discussed. Neoadjuvant radiotherapy is recommended for all high-grade (G2 and G3) tumors and was accompanied by intraoperative radiation when the STS was localized close to vital anatomic structures that might preclude achieving sufficient resection margins. Patients with chemosensitive tumors (rhabdomyosarcoma, synovial sarcoma, and extraosseous Ewing's sarcoma) were advised to receive neoadjuvant and adjuvant chemotherapy. Our approach to treatment and surgical strategy was extensively published previously ${ }^{25-33}$. Union for International Cancer Control (UICC) 7 th edition is used for staging, and Fédération Nationale des Centres de Lutte Contre Le Cancer (FNCLCC) classification is used for grading tumors.

To compare the groups and to analyze test accuracy, four-field tables were created. The groups were compared using Fisher's exact test. Wilcoxon signed-rank test was used to compare the time from biopsy to receiving a tumor board decision. The analysis was performed using Stata 14 (StataCorp LP, 4905 Lakeway Drive, College Station, TX 77845, USA), and results were considered significant if $p<0.05$. The Ethics committee of the Faculty of Medicine, University of Freiburg approved this study (282/19), and the need for informed consent was waived. All methods were carried out in accordance with relevant guidelines and regulations. The datasets are available from the corresponding author on request.

\section{Results}

Of 193 cancer patients included in the study, 91 had incisional biopsies and 102 CNB. 89/193 (46\%) of patients were female. The most common sarcoma subtype in our population was undifferentiated sarcoma (21\%), followed by liposarcoma (21\%), fibroblastic or myofibroblastic sarcomas (16\%), and liposarcoma (17\%) (excluding atypical lipomatous tumors, G1 liposarcoma). In the CNB group, we biopsied significantly more undifferentiated sarcomas and soft tissue metastases of other adenocarcinoma or non-solid tumors, whereas we found more sarcomas arising from vascular tissue in the incisional biopsy group. At the initial presentation, $79 \%$ of patients had no distant metastasis. The CNB population presented with a significantly higher rate of metastases. $60 \%$ of 


\begin{tabular}{|c|c|c|c|c|}
\hline & Incisional biopsy (\%) & Core needle biopsy (\%) & p-value & Total (\% in category) \\
\hline $\mathrm{N}$ & 91 & 102 & & 193 \\
\hline \multicolumn{5}{|l|}{ Gender } \\
\hline $\mathrm{m}$ & $49(54)$ & $57(56)$ & 0.88 & $104(54)$ \\
\hline $\mathrm{w}$ & $42(46)$ & $45(44)$ & & $89(46)$ \\
\hline Age $($ mean \pm SD) & $58.95 \pm 16.3$ & $61.32 \pm 18.48$ & 0.47 & \\
\hline \multicolumn{5}{|l|}{ Entity } \\
\hline Undifferentiated sarcoma & $11(12)$ & $30(29)$ & 0.0045 & $41(21)$ \\
\hline Liposarcoma $^{\pi}$ & $27(30)$ & $14(14)$ & 0.08 & $41(21)$ \\
\hline Fibroblastic/myofibroblastic sarcoma $^{+}$ & $20(22)$ & $10(10)$ & 0.028 & $30(16)$ \\
\hline $\begin{array}{l}\text { Vascular sarcoma (mainly angiosarcoma, but also hemangioendothelioma and Kaposi } \\
\text { sarcoma) }\end{array}$ & $11(12)$ & $2(2)$ & 0.007 & $13(7)$ \\
\hline Muscle sarcoma (rhabdomyosarcoma and leiomyosarcoma) & $5(5)$ & $2(2)$ & 0.25 & $7(4)$ \\
\hline Extraskeletal osteosarcoma and soft-tissue chondroma & $6(7)$ & $9(9)$ & 0.6 & $15(8)$ \\
\hline Synovial sarcoma & $6(7)$ & $4(4)$ & 0.52 & $10(5)$ \\
\hline Desmoid fibromatosis and myxoma & $5(5)$ & $5(5)$ & 1.00 & $10(5)$ \\
\hline Soft tissue metastasis (adenocarcinoma, non-solid tumors) & 0 & $26(25)$ & 0.0001 & $26(13)$ \\
\hline \multicolumn{5}{|l|}{ M-status } \\
\hline M0 & $85(93)$ & $68(67)$ & 0.001 & $153(79)$ \\
\hline M1 & $6(7)$ & $34(33)$ & & $40(21)$ \\
\hline \multicolumn{5}{|l|}{ Grading* } \\
\hline 3 & $28(51)$ & $42(68)$ & 0.03 & $70(60)$ \\
\hline 2 & $16(29)$ & $15(24)$ & & $31(26)$ \\
\hline 1 & $11(20)$ & $5(8)$ & & $16(14)$ \\
\hline \multicolumn{5}{|l|}{ NCCN stage } \\
\hline \multicolumn{5}{|l|}{ I } \\
\hline $\mathrm{A}$ & $3(4)$ & $2(5)$ & 0.22 & $5(4)$ \\
\hline $\mathrm{B}$ & $17(22)$ & $7(18)$ & & $24(21)$ \\
\hline \multicolumn{5}{|l|}{ II } \\
\hline $\mathrm{A}$ & $10(13)$ & $3(8)$ & & $13(11)$ \\
\hline $\mathrm{B}$ & $13(17)$ & $3(8)$ & & $16(14)$ \\
\hline III & $28(37)$ & $15(39)$ & & $43(38)$ \\
\hline IV & $5(7)$ & $8(21)$ & & $13(11)$ \\
\hline \multicolumn{5}{|l|}{ Radiotherapy } \\
\hline Neoadjuvant & $9(10)$ & $22(22)$ & 0.02 & 31 \\
\hline Intraoperative & $12(13)$ & $11(11)$ & 0.66 & \\
\hline Adjuvant & $32(35)$ & $11(11)$ & 0.001 & \\
\hline \multicolumn{5}{|l|}{ Chemotherapy } \\
\hline Neoadjuvant & $7(8)$ & $4(4)$ & 0.3 & \\
\hline Adjuvant & $5(5)$ & $3(3)$ & 0.47 & \\
\hline \multicolumn{5}{|l|}{ Anatomical distribution } \\
\hline Upper extremity & $16(18)$ & $16(15)$ & 0.37 & $32(17)$ \\
\hline Lower extremity & $59(65)$ & $57(56)$ & & $116(60)$ \\
\hline Trunk & $11(12)$ & $20(20)$ & & $31(16)$ \\
\hline Head and neck & $8(5)$ & $9(9)$ & & $14(7)$ \\
\hline
\end{tabular}

Table 1. Overview of patients included in the study. "Includes tumors classified as "round cell" before the 2013 Classification; ${ }^{+}$includes dermatofibrosarcoma protuberans, malignant solitary fibrous tumor, malignant fibrous histiocytoma (entity removed from the classification in the 2013 WHO Classification of Tumors of Soft Tissue); ${ }^{*}$ on biopsy, not attempted on all specimens, not reported for specimens that have undergone neoadjuvant irradiation, and not reported for non-sarcoma tumors.

patients had a G3 STS, 26\% a G2, and 14\% a G1 tumor, with CNB patients exhibiting significantly less differentiated (higher G) tumors. The distribution of NCCN stages, administration of chemotherapy, and anatomical distribution were even across both groups. CNB patients underwent neoadjuvant radiation therapy in more cases than patients diagnosed by incisional biopsy (Table 1 ).

Analysis of all patients regarding the accuracy of biopsy techniques revealed a $96 \%$ accuracy for diagnosing malignancy via CNB and $100 \%$ for incisional biopsy. Tumor subtype diagnosis was confirmed by pathology on the definitive specimen in $89 / 102(87 \%) \mathrm{CNB}$ cases and $81 / 91(89 \%)$ incisional biopsy cases $(p=0.52)$. Grading 


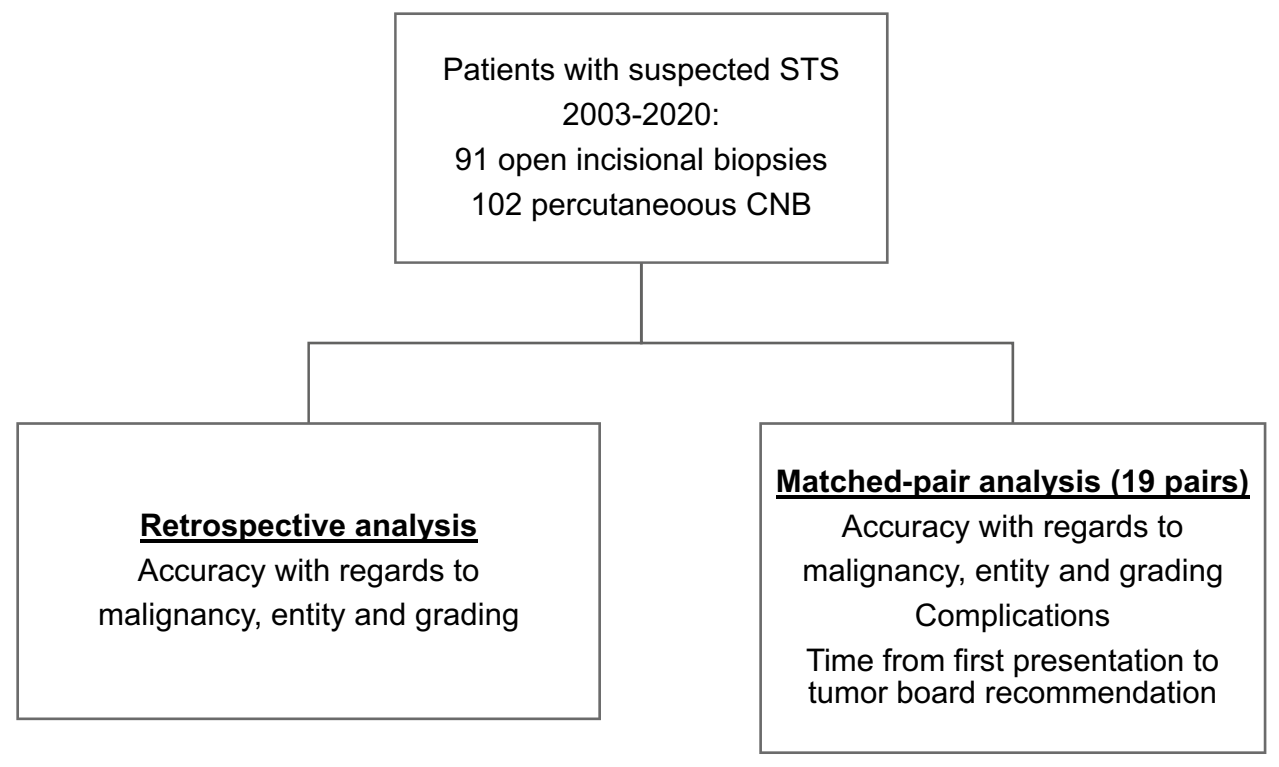

Figure 1. Patients with suspected soft tissue sarcoma (SFT) between 2003 and 2019 were included in the study. The core needle biopsy (CNB) was introduced in our department in July 2015, and since then, 102 patients have been diagnosed with CNB. These were compared to 91 open incisional biopsies regarding accuracy in diagnosing the correct entity and tumor grading. Secondly, a pair-matched analysis was conducted on 19 pairs, matching gender, age, and tumor characteristics: histology, localization, size, depth, and grading. The pairs were compared for sensitivity, specificity, the accuracy of diagnosis, complications, and time to case presentation in the interdisciplinary tumor board.

was correct in $54(87 \%)$ CNB specimens and 46 (84\%) incisional biopsy specimens $(p=0.61)$. When excluding non-sarcoma tumors from the analysis the accuracy of tumor subtype diagnosis was at $83 \%$ for CNB and $89 \%$ for IB $(p=0.25)$ and the accuracy of grading at $88 \%$ and $84 \%$, respectively $(p=0.59$, Table 2$)$.

For the matched-pair analysis, we created 19 patient pairs that matched according to gender, age, and tumor characteristics: entity, localization, size, depth, and grading. Matching of gender and tumor grading was possible in all pairs. A match in at least 5 of 7 tumor criteria was required to build a pair. Supplementary Table 1 gives an overview of the pairs. Sixteen of 19 cases had a G3,> T3 sarcoma of the lower extremity. The correct entity was diagnosed in nearly all cases, and the correct grading was diagnosed in $90 \%$ of incisional biopsies and $94 \%$ of CNBs $(p=0.62)$. The time from the initial consultation until case presentation and discussion in the interdisciplinary tumor board was significantly shorter for the CNB group ( $8.4 \pm 5$ days) compared to the incisional biopsy group $(15.6 \pm 6.6$ days, $p<0.002)$. Time from initial consultation to initiation of therapy (surgical or neoadjuvant) was also shorter, however not significantly ( $22.83 \pm 17.4$ vs. $28.83 \pm 18.6$ days, $p=0.32$ ). Time from biopsy to initiation of therapy was not different $(22.44 \pm 17.5$ vs. $24.11 \pm 17.5, p=0.77)$. Amongst 19 incisional biopsies, two wound infections and one hematoma requiring revision occurred. There was one wound infection after CNB (Table 3).

During the study period, the level of diagnostic accuracy regarding tumor subtype increased $\left(\mathrm{R}^{2}=0.02\right)$. Molecular diagnostics for sarcoma were introduced in our institution in 2011, and CNB in 2015 (Fig. 3). Interestingly, the use of molecular diagnostics did not increase diagnostic accuracy with regards to tumor subtype. Out of 61 cases in which molecular pathology was performed, the correct diagnosis was achieved in 54 (88\%) of cases. The same percentage of diagnostic accuracy was seen in patients in which molecular pathology was not used $(116$ out of $132,88 \%, p=1)$. The most common molecular tests performed in this series are listed in Table 4 . It is of clinical significance to ask whether the diagnostic discrepancy between CNB and the definitive specimen pathology influenced patient treatment. Table 5 lists these discrepancies. In one case, a more accurate tumor diagnosis might have resulted in recommending a different neoadjuvant therapy. We found most discrepancies in grading regarding the difference between G2 and G3 STS (6 cases). For most sarcoma entities, both G2 and G3 graded tumors underwent neoadjuvant radiation, rendering this discrepancy clinically inconsequential. Two cases had a discrepancy between G1 and G2, which prevented the recommendation for neoadjuvant radiation therapy in these cases.

Case presentations. We chose two clinical cases to illustrate the advantages and disadvantages of CNB. Figure 2 demonstrates the most common scenario of a tumor that is easily accessible to CNB. A 78-year old patient presented with a large, palpable mass in the left thigh. MRI showed a $15 \times 9 \mathrm{~cm}$ heterogeneous, contrastenhanced tumor (A and B), which we biopsied using a HistoCore core needle biopsy device (C and D). One day after the initial consultation, the histological workup revealed the diagnosis of high-grade sarcoma. Additional immunohistochemistry specified the diagnosis of an undifferentiated pleomorphic sarcoma five days following biopsy, enabling the interdisciplinary discussion in the tumor board seven days after the initial consultation. 

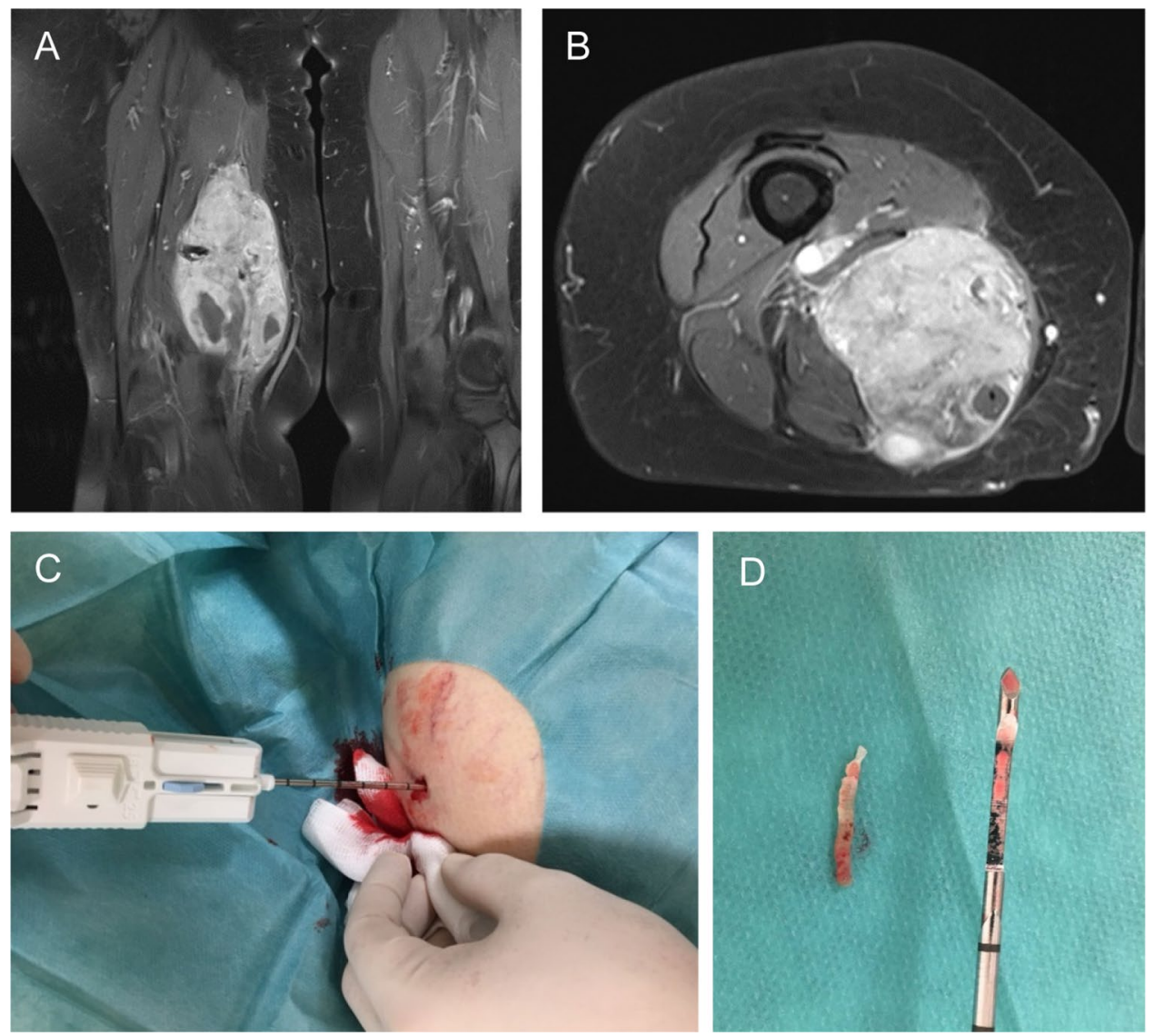

Figure 2. A 78-year old patient was referred to us with a large, palpable mass in the left leg. An MRI showed a $15 \times 9 \mathrm{~cm}$ heterogeneous, contrast-enhanced tumor $(\mathbf{A}, \mathbf{B})$, which was biopsied using a HistoCore core needle biopsy device (C,D). One day after the initial consultation, the diagnosis of a high-grade sarcoma was made by the pathologist. Additional immunohistochemistry specified the diagnosis of an undifferentiated pleomorphic sarcoma five days following biopsy, enabling the interdisciplinary discussion in the tumor board seven days after the initial consultation.

\begin{tabular}{|l|l|l|l|}
\hline \multirow{2}{*}{} & \multicolumn{2}{|l|}{ Biopsy result } & \multirow{2}{*}{$\boldsymbol{P}$-value } \\
\cline { 2 - 3 } & CNB (\%) N=102 & IB (\%) N=91 & 0.91 \\
\hline Malignancy & $98(96)$ & $92(100)$ & 0.52 \\
\hline Tumor subtype & $89(87)$ & $81(89)$ & 0.19 \\
\hline Grading & $81(91)$ & $46(84)$ & \\
\hline Accuracy of biopsy excluding metastases and other non-sarcoma tumors & $81(89)$ & 0.25 \\
\hline Tumor subtype & $63(83)$ & $46(84)$ & 0.59 \\
\hline Grading & $53(88)$ & & \multicolumn{2}{|l}{} \\
\hline
\end{tabular}

Table 2. Analysis of all patients $(\mathrm{N}=193)$ reporting the accuracy of incisional and core needle biopsies with regards to diagnosis of malignancy and accuracy in diagnosing entity and grading. Accuracy is defined as agreement between pathology results on biopsy tissue and on the surgical resection specimen. Grading was diagnosed in 89 CNB and 55 incisional biopsy cases. Since the CNB group included 26 cases of non-sarcoma tumors, the accuracy of biopsies was separately calculated excluding these tumors. $C N B$ core needle biopsy; $I B$ incisional biopsy.

Figure 4 shows coronal and axial magnetic resonance images of a heterogeneous contrast-enhanced tumor of the lower leg in a 19-year-old girl. Here, two percutaneous CNBs from the posterior region of the tumor were attempted (red arrow in B) because the ventral part of the tumor had a close relationship to the peroneal nerve. Both biopsies resulted in inconclusive pathology. Thus, we performed an open biopsy from a more contrastenhancing ventral region (green arrow in B) to diagnose extraskeletal Ewing's sarcoma. The estimated delay to 


\begin{tabular}{|l|l|l|l|}
\hline & Incisional biopsy & Core needle biopsy & p \\
\hline Sensitivity (malignancy) & $100 \%$ & $100 \%$ & \\
\hline Positive predictive value (malignancy) & $100 \%$ & $100 \%$ & \\
\hline Correct entity & $96 \%$ & $98 \%$ & n.s \\
\hline Correct grading & $90 \%$ & $94 \%$ & 0.62 \\
\hline $\begin{array}{l}\text { Time from initial consultation to case presentation } \\
\text { in tumor board (days) }\end{array}$ & $15.6 \pm 6.6$ & $8.4 \pm 5$ & $<0.002$ \\
\hline $\begin{array}{l}\text { Time from initial consultation to initiation of } \\
\text { therapy* (days) }\end{array}$ & $28.83 \pm 18.6$ & $22.83 \pm 17.4$ & 0.32 \\
\hline Time from biopsy to initiation of therapy* (days) & $24.11 \pm 17.5$ & $22.44 \pm 17.5$ & 0.77 \\
\hline $\begin{array}{l}\text { Time from biopsy to case presentation in tumor } \\
\text { board (days) }\end{array}$ & $15.6 \pm 6.6$ & $8.4 \pm 5$ & $<0.002$ \\
\hline Anesthesia & 15 general & 19 local & \\
\hline Complications & 2 wound infections 1 hematoma requiring revision & 1 wound infection & \\
\hline
\end{tabular}

Table 3. Results of the pair-matched analysis on 19 pairs matched according to gender, age, and tumor characteristics: entity, localization, size, depth, and grading. Sixteen of 19 cases had a G3, > T3 sarcoma of the lower extremity. ${ }^{*}$ Surgery, or beginning of neoadjuvant chemotherapy or radiation therapy.

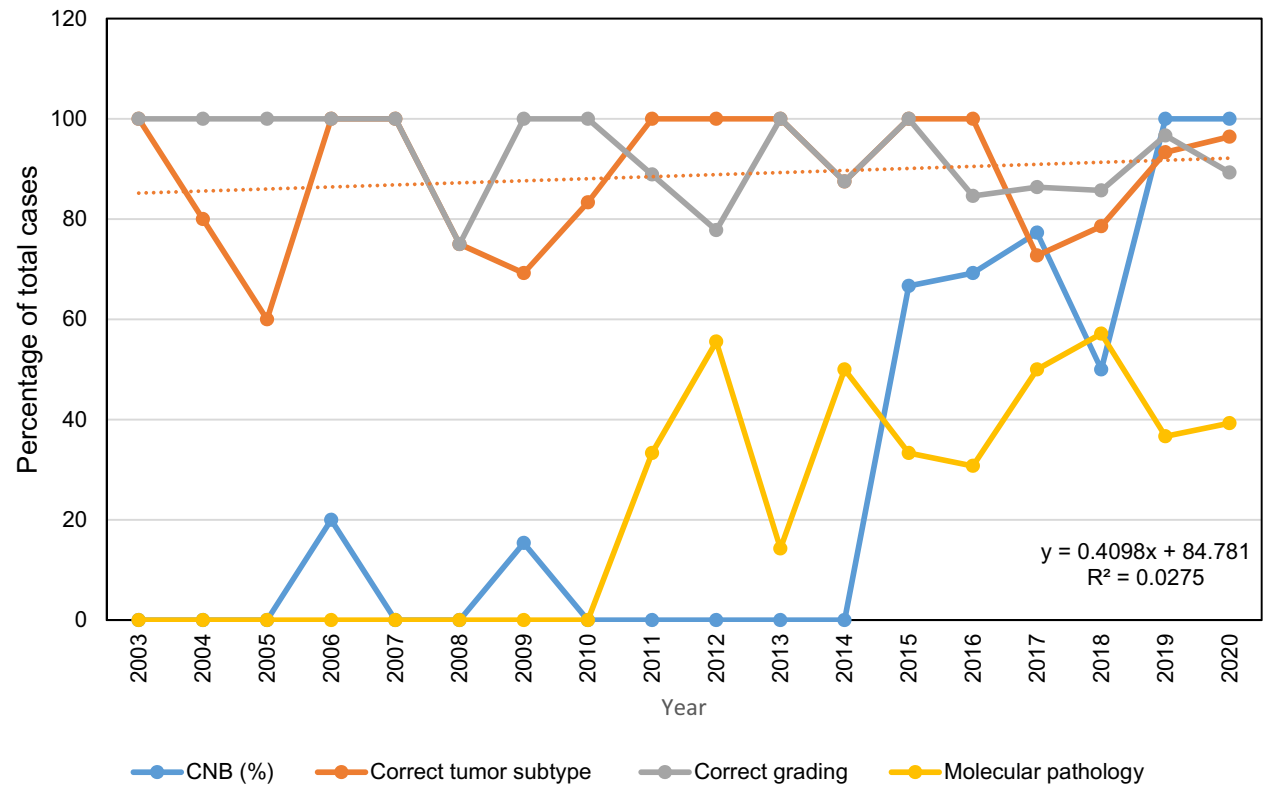

Figure 3. The level of diagnostic accuracy with regards to tumor type increased during the study period $\left(\mathrm{R}^{2}=0.02\right)$. Results for tumor accuracy have been fitted to create the regression line. An increase in molecular pathological testing is noted beginning in 2011, and an increase in the use of core needle biopsy (CNB) is noted from 2015.

diagnosis from the two failed CNBs, in this case, was two and a half weeks. In this case, persistence in acquiring the diagnosis was essential, as a negative CNB result does not exclude malignancy.

Apart from this case, CNB failed to diagnose malignancy in three other cases in this series. In these cases, imaging was strongly suggestive of undifferentiated sarcoma, and biopsy showed necrotic tissue, also strongly suggestive of malignancy. Therefore there was no need for new biopsy and primary surgery has been suggested, so that there was no delay in treatment.

\section{Discussion}

This retrospective analysis provides evidence that core needle biopsy is as accurate as incisional biopsy in diagnosing soft tissue sarcoma. CNB achieved histological accuracy for subtype diagnosis in $87 \%$ of cases compared to $89 \%$ from incisional biopsy. As confirmed by pathology on the definitive specimen, tumor grading was correct in $87 \%$ of CNB specimens compared to $84 \%$ of incisional biopsy specimens. In addition, CNB led to faster diagnosis of malignancy while being less burdensome for patients and clinical resources. Performing CNB led to a shorter time between the initial presentation and tumor board decision than performing IB. Time from the initial presentation and initiation of therapy was also shorter in the CNB group, but not significantly. 


\begin{tabular}{|l|l|l|l|l|l|}
\hline Gene product & Sarcoma subtype & Affected pathway & Incisional biopsy & Core needle biopsy & Total \\
\hline MDM2 & Liposarcoma & Lack of function of the p53 tumor suppressor & 12 & 3 \\
\hline CDK4 & Liposarcoma & Cyclin-dependent kinase & 14 & 15 \\
\hline DDIT3 & Myxoid/round cell liposarcoma & $\begin{array}{l}\text { CCAAT/enhancer-binding protein family (pro-apoptotic tran- } \\
\text { scription factors) }\end{array}$ & 12 & 3 \\
\hline SS18 & Synovial sarcoma & SWI/SNF chromatin remodeling complex & 7 & 7 \\
\hline EWSR family members & Ewing sarcoma & FET family of transcriptional regulators & 8 & 5 & 17 \\
\hline GNAS & Intramuscular myxoma & Activation of cAMP-Dependent protein kinase A & 1 & 19 \\
\hline CTNNB1 & Desmoid tumors & $\begin{array}{l}\text { Beta-catenin (regulation of cell-cell adhesion and gene transcrip- } \\
\text { tion) }\end{array}$ & 1 & 6 & 2 \\
\hline RNA fusion pannel & & & 8 & 12 & 0 \\
\hline Others* & & & 3 & 2 \\
\hline
\end{tabular}

Table 4. Overview of genes targeted by molecular diagnostics in this study. ${ }^{\star} \mathrm{EBV}, \mathrm{BCL}$ family members, Myc, USP6.

\begin{tabular}{|c|c|c|c|c|c|}
\hline Patient & Age & Localization & CNB diagnosis & Final diagnosis & Influence on treatment \\
\hline 1 & 58 & Shoulder & Myxoid liposarcoma & Myxoid chondro-sarcoma & $\begin{array}{l}\text { Yes, neoadjuvant chemotherapy would likely have been proposed for chondro- } \\
\text { sarcoma }\end{array}$ \\
\hline 2 & 70 & Abdomen & Pleomorphic sarcoma & Inflammatory fibrocystic histiocytoma & No \\
\hline 3 & 85 & Thigh & Myxoid sarcoma & Pleomorphic sarcoma & No \\
\hline 4 & 78 & Forearm & Myxofibrosar-coma & Pleomorphic spindle cell sarcoma & No \\
\hline
\end{tabular}

Table 5. In 13 patients, the final diagnosis differed from the core needle biopsy (CNB) diagnosis. In four patients, CNB failed to diagnose malignancy. In two patients, diagnosis of the malignant tumor was achieved, but not the subtype. In four cases, subtype diagnosis was attempted but differed from the definitive diagnosis. The latter four cases are listed here, reflecting whether the incorrect initial diagnosis influenced the treatment. This seems to have been the case in only one patient.
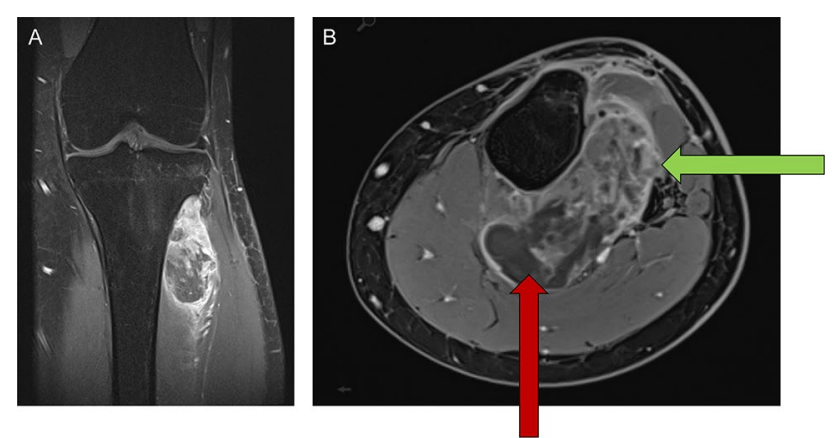

Figure 4. Coronal and axial magnetic resonance images showing a heterogeneous contrast-enhanced tumor of the leg in a 19-year-old girl. Two percutaneous CNB from the posterior region of the tumor were performed (red arrow in B) because the ventral part of the tumor had a close relationship to the peroneal nerve. Both biopsies resulted in inconclusive pathology. An open biopsy was required from a more contrast-enhancing ventral region (green arrow in B) to diagnose extraskeletal Ewing's sarcoma. The estimated delay to diagnosis from the two failed CNBs, in this case, was two and a half weeks. This case illustrates the importance of persistence in acquiring the diagnosis as a negative $\mathrm{CNB}$ result does not exclude malignancy. The ability to correlate clinical, radiological, and pathological findings is crucial.

During the last decade, reports have been accumulating on the reliability of CNB for the primary diagnosis of soft tissue tumors ${ }^{13-22}$. A recent meta-analysis including 17 studies and 2680 patients confirmed that CNB has high accuracy in diagnosing the lesions with fewer complications than incisional biopsy ${ }^{23}$. Thus, in many centers, including ours, CNB is the primary method for biopsy soft tissue tumors. While the ability to diagnose malignancy by CNB is widely accepted, several authors have reported varying accuracy levels in diagnosing the correct tumor entity and grading ${ }^{7-12}$. Concerning the competing method of fine-needle aspiration biopsy, there is widespread consensus, with very few exceptions ${ }^{34}$, that it is significantly less accurate and, thus, inferior to both open incisional biopsy and $\mathrm{CNB}^{35,36}$. 
As the matched-pair analysis demonstrated, the most typical case encountered in a soft tissue tumor center is a patient with a large, palpable mass of the thigh and an MRI suggestive of malignancy (Fig. 2). These tumors are easily accessible by CNB, and the matched-pair analysis showed a $100 \%$ sensitivity for malignancy and a $>95 \%$ correct diagnosis of the entity in both biopsy techniques. With almost $90 \%$ correct entities in the analysis of all patients, our study exhibits a relatively high degree of diagnostic accuracy based on either type of biopsy material. The diagnostic accuracy increased during the study period. In the literature, accuracy regarding the histological tumor type has been reported as high as $83 \%{ }^{16}, 80 \%{ }^{13}$ or even as low as $45 \%{ }^{6}$, and, in two recent large meta-analyses, at $84 \%^{37}$ and $88 \%{ }^{23}$. Diagnostic accuracy in sarcoma biopsy is multifactorial. We consider the existence of dedicated specialists from every department involved, who stay in close communication with each other, to be of utmost importance for accurate and efficient sarcoma diagnosis. The radiologist and the surgeon need to have sufficient experience in sarcoma diagnosis and treatment to precisely determine which portion of the tumor is most likely to yield significant histologic information. Execution of the biopsy involves precision and critical judgment. Possibly, soft tissue tumors are even better sampled by CNB than incisional biopsy, since $\mathrm{CNB}$ allows for fanning out and sampling deeper parts of the mass. We require at least three passes of the needle per tumor (the mean was $3.59 \pm 2$ in this study), and this amount of tissue is sufficient for all pathological tests ${ }^{38}$. We thus consider the increasing expertise of the interdisciplinary team to be the main factor for the increase in accuracy of the histological diagnosis seen in our analysis. CNB can be performed under ultrasound guidance if needed, but we do not consider computer tomography (CT) or MRI guidance necessary, despite several positive series in the literature ${ }^{39-41}$.

Molecular diagnostics are the most exciting recent development in the sarcoma field. Increased understanding of molecular mechanisms of sarcoma allows us to reconsider the classification system, implying potential clinical consequences ${ }^{4}$. The most commonly investigated gene products were MDM2 and CDK ${ }^{42,43}$, the mutations of which are markers for liposarcoma. This is followed by DDIT3, mutated in myxoid sarcoma, SS18 (synovial sarcoma $^{44}$ ), EWSR family members (Ewing sarcoma ${ }^{45}$ ), and GNAS and CTNNB1 that are mutated in intramuscular myxoma and desmoid fibromatosis ${ }^{46}$, respectively (Table 4$)$.

A prospective multicenter study on 395 patients has demonstrated that molecular diagnostics can change diagnosis in specific STS subtypes ${ }^{43,47,48}$. The authors have cited several tumors that were reclassified as dermatofibrosarcoma protuberans, requiring surgery to reach R0 status, and several cases that were reclassified from the initial diagnosis of Ewing sarcoma, thus sparing these patients from useless chemotherapy. In our series, there were no cases where a change in therapy was affected by molecular pathology findings. For example, the most common molecular tests for MDM2 and CDK4 mutations were used to dedifferentiated liposarcoma and undifferentiated pleomorphic sarcoma, and both entities are treated identically. However, there are suggestions that MDM2 negative undifferentiated pleomorphic sarcomas have a less favorable outcome than MDM2 positive tumors $^{42,43}$. Molecular diagnostics were instrumental in diagnosing desmoid fibromatosis and intramuscular myxoma, as these tumor entities are notoriously challenging to diagnose, but the exact diagnosis does not change the course of therapy. In certain situations, such as differentiation between melanoma and peripheral nerve sheet tumors, molecular diagnostics are essential. After correlating the use of molecular diagnostics and diagnostic accuracy, we observed that molecular diagnostic methods were not associated with higher diagnostic accuracy in this study. Still, we agree that molecular diagnostics should be mandatory for sarcoma diagnosis. In our institution, in-situ hybridization is completed within 1-2 weeks. Thus, there is no delay in therapy. Fusion panels can take up to 4-6 weeks, and they are mostly needed to differentiate non-malignant tumors and low-grade sarcomas.

Future developments in molecular diagnostics will open new avenues of diagnostic and therapeutic possibilities. For instance, we $\mathrm{e}^{49-51}$ and others ${ }^{52,53}$ explore recurrent sarcoma translocations as targets for "liquid biopsy." Here, circulating DNA harboring a specific translocation is measured to monitor tumor activity, which has relevance for patient follow-up and assessing the success of multimodal sarcoma therapy. Further developments are expected in the area of targeted molecular therapeutics, which will benefit patients with advanced sarcoma ${ }^{54}$.

In our study, the discrepancy in diagnosis and grading based on definitive resection specimen was, in most cases, not relevant for therapy. Still, there were some cases in which therapy would have been different. For instance, some patients with high-grade sarcomas might benefit from neoadjuvant irradiation, which is not recommended if the biopsy suggests a lower tumor grading (Table 5). It is crucial to discuss the few cases in which CNB failed to diagnose malignancy, requiring incisional biopsy (Fig. 4). A negative CNB does not ensure the absence of malignancy. The ability to correlate clinical, radiological, and pathological findings is crucial, and the clinicians have to persist in achieving the correct diagnosis. Furthermore, it is paramount to place the biopsy in the area of future resection to avoid contaminating healthy tissues with tumor cells and impede tumor resection. For all these reasons, tumor biopsy is not delegable, and the surgeon who will perform the tumor resection needs to perform the biopsy.

Our study included non-malignant tumors relevant to differential diagnosis, such as desmoid tumors and intramuscular myxoma, but excluded lipomas. Looking into differentiation by biopsy between lipomas and atypical lipomatous tumors is an interesting question but one we decided not to investigate here because it does not fit the scope of the present work. At the beginning of the study period, in 2013, a change in sarcoma classification took place ${ }^{3}$. We thus had to classify previously used entities, such as malignant fibrous histiocytoma, in broader categories (see explanation to Table 1). The changes in sarcoma classification did not influence the statistical analysis of the test sensitivity in this study, as each biopsy was compared to the resection specimen, which was diagnosed according to the same classification. The CNB group in this work harbors more advanced and more aggressive tumors (higher rate of metastases and higher grading). This is due to the temporal development in our institution since, with time, our regional reputation as sarcoma center grew, resulting in more advanced cases presenting to us. This difference also has no significance for the interpretation of the current research question.

In light of our data and the pertinent literature, we conclude that CNB is reliable and safe as a method of the first choice for soft tissue sarcoma biopsy. It has the same diagnostic accuracy as incisional biopsy, and the speed 
and convenience of the method greatly exceeded those of incisional biopsy. However, both its reliability and safety depend on the expertise and high degree of specialization of the surgeon and the pathologist.

Received: 14 September 2021; Accepted: 14 January 2022

Published online: 03 February 2022

\section{References}

1. Lahat, G., Lazar, A. \& Lev, D. Sarcoma epidemiology and etiology: potential environmental and genetic factors. Surg. Clin. N. Am. 88, 451-481. https://doi.org/10.1016/j.suc.2008.03.006 (2008).

2. Sbaraglia, M., Bellan, E. \& Dei Tos, A. P. The 2020 WHO classification of soft tissue tumours: News and perspectives. Pathologica https://doi.org/10.32074/1591-951X-213 (2020).

3. Doyle, L. A. Sarcoma classification: An update based on the 2013 World Health Organization Classification of Tumors of Soft Tissue and Bone. Cancer 120, 1763-1774. https://doi.org/10.1002/cncr.28657 (2014).

4. Schaefer, I. M. \& Fletcher, C. D. M. Recent advances in the diagnosis of soft tissue tumours. Pathology 50, 37-48. https://doi.org/ 10.1016/j.pathol.2017.07.007 (2018).

5. Marino-Enriquez, A. \& Bovee, J. V. Molecular pathogenesis and diagnostic, prognostic and predictive molecular markers in sarcoma. Surg. Pathol. Clin. 9, 457-473. https://doi.org/10.1016/j.path.2016.04.009 (2016).

6. Kasraeian, S., Allison, D. C., Ahlmann, E. R., Fedenko, A. N. \& Menendez, L. R. A comparison of fine-needle aspiration, core biopsy, and surgical biopsy in the diagnosis of extremity soft tissue masses. Clin. Orthop. Relat. Res. 468, 2992-3002. https://doi. org/10.1007/s11999-010-1401-x (2010).

7. Skrzynski, M. C., Biermann, J. S., Montag, A. \& Simon, M. A. Diagnostic accuracy and charge-savings of outpatient core needle biopsy compared with open biopsy of musculoskeletal tumors. J, Bone Jt. Surg. 78, 644-649 (1996). https://doi.org/10.2106/00004 623-199605000-00002

8. Rougraff, B. T., Aboulafia, A., Biermann, J. S. \& Healey, J. Biopsy of soft tissue masses: evidence-based medicine for the musculoskeletal tumor society. Clin. Orthop. Relat. Res. 467, 2783-2791. https://doi.org/10.1007/s11999-009-0965-9 (2009).

9. Pohlig, F. et al. Percutaneous core needle biopsy versus open biopsy in diagnostics of bone and soft tissue sarcoma: A retrospective study. Eur. J. Med. Res. 17, 29. https://doi.org/10.1186/2047-783X-17-29 (2012).

10. Ogilvie, C. M., Torbert, J. T., Finstein, J. L., Fox, E. J. \& Lackman, R. D. Clinical utility of percutaneous biopsies of musculoskeletal tumors. Clin. Orthop. Relat. Res. 450, 95-100. https://doi.org/10.1097/01.blo.0000229302.52147.c7 (2006).

11. Kissin, M. W., Fisher, C., Carter, R. L., Horton, L. W. \& Westbury, G. Value of Tru-cut biopsy in the diagnosis of soft tissue tumours. Br. J. Surg. 73, 742-744. https://doi.org/10.1002/bjs.1800730921 (1986).

12. Heslin, M. J., Lewis, J. J., Woodruff, J. M. \& Brennan, M. F. Core needle biopsy for diagnosis of extremity soft tissue sarcoma. Ann. Surg. Oncol. 4, 425-431. https://doi.org/10.1007/BF02305557 (1997).

13. Hoeber, I., Spillane, A. J., Fisher, C. \& Thomas, J. M. Accuracy of biopsy techniques for limb and limb girdle soft tissue tumors. Ann. Surg. Oncol. 8, 80-87. https://doi.org/10.1007/s10434-001-0080-y (2001).

14. Ball, A. B., Fisher, C., Pittam, M., Watkins, R. M. \& Westbury, G. Diagnosis of soft tissue tumours by Tru-Cut biopsy. Br. J. Surg. 77, 756-758. https://doi.org/10.1002/bjs.1800770713 (1990).

15. Adams, S. C., Potter, B. K., Pitcher, D. J. \& Temple, H. T. Office-based core needle biopsy of bone and soft tissue malignancies: an accurate alternative to open biopsy with infrequent complications. Clin. Orthop. Relat. Res. 468, 2774-2780. https://doi.org/10. 1007/s11999-010-1422-5 (2010).

16. Woon, D. T. \& Serpell, J. W. Preoperative core biopsy of soft tissue tumours facilitates their surgical management: a 10-year update. ANZ J. Surg. 78, 977-981. https://doi.org/10.1111/j.1445-2197.2008.04715.x (2008).

17. Welker, J. A., Henshaw, R. M., Jelinek, J., Shmookler, B. M. \& Malawer, M. M. The percutaneous needle biopsy is safe and recommended in the diagnosis of musculoskeletal masses. Cancer 89, 2677-2686. https://doi.org/10.1002/1097-0142(20001215)89:12\% 3c2677::aid-cncr22\%3e3.0.co;2-1 (2000).

18. Walker, J. B., Stockwell, E., Worhacz, K., Kang, P. \& Decomas, A. Safety and accuracy of core needle biopsy for soft tissue masses in an ambulatory setting. Sarcoma 2018, 1657864. https://doi.org/10.1155/2018/1657864 (2018).

19. Strauss, D. C. et al. The role of core needle biopsy in the diagnosis of suspected soft tissue tumours. J. Surg. Oncol. 102, 523-529. https://doi.org/10.1002/jso.21600 (2010).

20. Srisawat, P. et al. Comparative study of diagnostic accuracy between office-based closed needle biopsy and open incisional biopsy in patients with musculoskeletal sarcomas. J. Med. Assoc. Thai. 97(Suppl 2), S30-38 (2014).

21. Mitsuyoshi, G. et al. Accurate diagnosis of musculoskeletal lesions by core needle biopsy. J. Surg. Oncol. 94, 21-27. https://doi.org/ $10.1002 /$ jso.20504 (2006).

22. Ray-Coquard, I. et al. Evaluation of core needle biopsy as a substitute to open biopsy in the diagnosis of soft-tissue masses. Eur. J. Cancer 39, 2021-2025. https://doi.org/10.1016/s0959-8049(03)00430-1 (2003).

23. Birgin, E. et al. Core needle biopsy versus incisional biopsy for differentiation of soft-tissue sarcomas: A systematic review and meta-analysis. Cancer 126, 1917-1928. https://doi.org/10.1002/cncr.32735 (2020).

24. von Mehren, M. et al. Soft tissue sarcoma, Version 2.2018, NCCN Clinical Practice Guidelines in Oncology. J. Natl. Compr. Cancer Netw. 16, 536-563 (2018). https://doi.org/10.6004/jnccn.2018.0025.

25. Bannasch, H. et al. The diagnosis and treatment of soft tissue sarcomas of the limbs. Deutsches Arzteblatt international 108, 32-38. https://doi.org/10.3238/arztebl.2011.0032 (2011).

26. Bannasch, H., Haivas, I., Momeni, A. \& Stark, G. B. Oncosurgical and reconstructive concepts in the treatment of soft tissue sarcomas: a retrospective analysis. Arch. Orthop. Trauma Surg. 129, 43-49. https://doi.org/10.1007/s00402-008-0576-z (2009).

27. Koulaxouzidis, G., Schwarzkopf, E., Bannasch, H. \& Stark, G. B. Is revisional surgery mandatory when an unexpected sarcoma diagnosis is made following primary surgery?. World J. Surg. Oncol. 13, 306. https://doi.org/10.1186/s12957-015-0719-y (2015).

28. Simunovic, F. et al. Microsurgical reconstruction of oncological scalp defects in the elderly. J. Plast. Reconstr. Aesthet. Surg. 69, 912-919. https://doi.org/10.1016/j.bjps.2016.03.021 (2016).

29. Simunovic, F. et al. The use of deep inferior epigastric vessels to revascularise free flaps for reconstruction of complex oncosurgical defects of the thigh and the abdomen. Handchir Mikrochir Plast Chir 50, 259-268. https://doi.org/10.1055/s-0043-124187 (2018).

30. Zeller, J. et al. Efficacy and safety of microsurgery in interdisciplinary treatment of sarcoma affecting the bone. Front. Oncol. 9, 1300. https://doi.org/10.3389/fonc.2019.01300 (2019).

31. Bach, A. D., Kopp, J., Stark, G. B. \& Horch, R. E. The versatility of the free osteocutaneous fibula flap in the reconstruction of extremities after sarcoma resection. World J. Surg. Oncol. 2, 22. https://doi.org/10.1186/1477-7819-2-22 (2004).

32. Koulaxouzidis, G., Simunovic, F. \& Bannasch, H. Soft tissue sarcomas of the arm oncosurgical and reconstructive principles within a multimodal, interdisciplinary setting. Front. Surg. 3, 12. https://doi.org/10.3389/fsurg.2016.00012 (2016).

33. Penna, V., Iblher, N., Momeni, A., Stark, G. B. \& Bannasch, H. Free tissue transfer in reconstruction following soft tissue sarcoma resection. Microsurgery 31, 434-440. https://doi.org/10.1002/micr.20895 (2011). 
34. Ogun, G. O. Fine needle Aspiration Biopsy (FNAB) in the initial evaluation and diagnosis of palpable soft tissue lesions and with histologic correlation. Pan. Afr. Med. J. 20, 44. https://doi.org/10.11604/pamj.2015.20.44.4271 (2015).

35. Dupuy, D. E., Rosenberg, A. E., Punyaratabandhu, T., Tan, M. H. \& Mankin, H. J. Accuracy of CT-guided needle biopsy of musculoskeletal neoplasms. AJR Am. J. Roentgenol. 171, 759-762. https://doi.org/10.2214/ajr.171.3.ajronline_171_3_001 (1998).

36. Yang, Y. J. \& Damron, T. A. Comparison of needle core biopsy and fine-needle aspiration for diagnostic accuracy in musculoskeletal lesions. Arch. Pathol. Lab. Med. 128, 759-764. https://doi.org/10.1043/1543-2165(2004)128\%3c759:CONCBA\%3e2.0.CO;2 (2004).

37. Kubo, T. et al. A meta-analysis supports core needle biopsy by radiologists for better histological diagnosis in soft tissue and bone sarcomas. Medicine Baltimore 97, e11567. https://doi.org/10.1097/MD.0000000000011567 (2018).

38. Agaimy, A. Value of core needle biopsy in preoperative diagnostics of soft tissue tumors: possibilities and limitations. Pathologe 35(Suppl 2), 189-197. https://doi.org/10.1007/s00292-014-2010-3 (2014).

39. Kiatisevi, P. et al. Computed tomography-guided core needle biopsy versus incisional biopsy in diagnosing musculoskeletal lesions. J. Orthop. Surg. (Hong Kong) 21, 204-208. https://doi.org/10.1177/230949901302100218 (2013).

40. Noebauer-Huhmann, I. M. et al. Use of diagnostic dynamic contrast-enhanced (DCE)-MRI for targeting of soft tissue tumour biopsies at 3T: preliminary results. Eur. Radiol. 25, 2041-2048. https://doi.org/10.1007/s00330-014-3576-0 (2015).

41. Issakov, J. et al. Computed tomography-guided core needle biopsy for bone and soft tissue tumors. Isr. Med. Assoc. J. 5, 28-30 (2003).

42. Le Guellec, S. et al. Are peripheral purely undifferentiated pleomorphic sarcomas with MDM2 amplification dedifferentiated liposarcomas?. Am. J. Surg. Pathol. 38, 293-304. https://doi.org/10.1097/PAS.0000000000000131 (2014).

43. Coindre, J. M. et al. Inflammatory malignant fibrous histiocytomas and dedifferentiated liposarcomas: Histological review, genomic profile, and MDM2 and CDK4 status favour a single entity. J. Pathol. 203, 822-830. https://doi.org/10.1002/path.1579 (2004).

44. Guillou, L. et al. Detection of the synovial sarcoma translocation $\mathrm{t}(\mathrm{X} ; 18)$ (SYT;SSX) in paraffin-embedded tissues using reverse transcriptase-polymerase chain reaction: a reliable and powerful diagnostic tool for pathologists. A molecular analysis of 221 mesenchymal tumors fixed in different fixatives. Human pathology 32, 105-112. https://doi.org/10.1053/hupa.2001.21130 (2001).

45. Brenca, M. et al. NR4A3 fusion proteins trigger an axon guidance switch that marks the difference between EWSR1 and TAF15 translocated extraskeletal myxoid chondrosarcomas. J. Pathol. 249, 90-101. https://doi.org/10.1002/path.5284 (2019).

46. Timbergen, M. J. M. et al. The prognostic role of beta-catenin mutations in desmoid-type fibromatosis undergoing resection only: A meta-analysis of individual patient data. Ann. Surg. 273, 1094-1101. https://doi.org/10.1097/SLA.0000000000003698 (2021).

47. Italiano, A. et al. Clinical effect of molecular methods in sarcoma diagnosis (GENSARC): A prospective, multicentre, observational study. Lancet Oncol 17, 532-538. https://doi.org/10.1016/S1470-2045(15)00583-5 (2016).

48. Weaver, J. et al. Fluorescence in situ hybridization for MDM2 gene amplification as a diagnostic tool in lipomatous neoplasms. Mod. Pathol. 21, 943-949. https://doi.org/10.1038/modpathol.2008.84 (2008).

49. Braig, D. et al. Genotyping of circulating cell-free DNA enables noninvasive tumor detection in myxoid liposarcomas. Int. J. Cancer 145, 1148-1161. https://doi.org/10.1002/ijc.32216 (2019).

50. Fricke, A. et al. Synovial sarcoma microvesicles harbor the SYT-SSX fusion gene transcript: Comparison of different methods of detection and implications in biomarker research. Stem Cells Int. 2016, 6146047. https://doi.org/10.1155/2016/6146047 (2016).

51. Fricke, A. et al. Identification of a blood-borne miRNA signature of synovial sarcoma. Mol. Cancer 14, 151. https://doi.org/10. 1186/s12943-015-0424-z (2015).

52. Hadjimichael, A. C., Pergaris, A., Kaspiris, A., Foukas, A. F. \& Theocharis, S. E. Liquid biopsy: A new translational diagnostic and monitoring tool for musculoskeletal tumors. Int. J. Mol. Sci. https://doi.org/10.3390/ijms222111526 (2021).

53. van der Laan, P., van Houdt, W. J., van den Broek, D., Steeghs, N. \& van der Graaf, W. T. A. Liquid biopsies in sarcoma clinical practice: Where do we stand? Biomedicines. https://doi.org/10.3390/biomedicines9101315 (2021).

54. Yuan, J., Li, X. \& Yu, S. Molecular targeted therapy for advanced or metastatic soft tissue sarcoma. Cancer Control 28, 10732748211038424. https://doi.org/10.1177/10732748211038424 (2021).

55. Sung, K. S., Seo, S. W. \& Shon, M. S. The diagnostic value of needle biopsy for musculoskeletal lesions. Int. Orthop. 33, $1701-1706$. https://doi.org/10.1007/s00264-009-0835-9 (2009).

56. Racanelli, D. et al. Next-generation sequencing approaches for the identification of pathognomonic fusion transcripts in sarcomas: The experience of the Italian ACC Sarcoma Working Group. Front. Oncol. 10, 489. https://doi.org/10.3389/fonc.2020.00489 (2020).

57. Jo, V. Y. \& Fletcher, C. D. WHO classification of soft tissue tumours: An update based on the 2013 (4th) edition. Pathology 46, 95-104. https://doi.org/10.1097/PAT.0000000000000050 (2014).

\section{Author contributions}

J.K., M.M., G.B.S., H.B., and F.S. conceived the project and formulated the research question. J.K., G.B.S., H.B., Ph.K., and F.S. treated the patients and performed the procedures described. J.K., M.M., Ph.K., and F.S. collected and analyzed the data. J.K., M.M., Ph.K., and F.S. created the figures and the tables. J.K., M.M., G.B.S., Ph.K., H.B., and F.S. wrote the manuscript.

\section{Funding}

Open Access funding enabled and organized by Projekt DEAL.

\section{Competing interests}

The authors declare no competing interests.

\section{Additional information}

Supplementary Information The online version contains supplementary material available at https://doi.org/ 10.1038/s41598-022-05752-4.

Correspondence and requests for materials should be addressed to F.S.

Reprints and permissions information is available at www.nature.com/reprints.

Publisher's note Springer Nature remains neutral with regard to jurisdictional claims in published maps and institutional affiliations. 
(c) (i) Open Access This article is licensed under a Creative Commons Attribution 4.0 International cc) License, which permits use, sharing, adaptation, distribution and reproduction in any medium or format, as long as you give appropriate credit to the original author(s) and the source, provide a link to the Creative Commons licence, and indicate if changes were made. The images or other third party material in this article are included in the article's Creative Commons licence, unless indicated otherwise in a credit line to the material. If material is not included in the article's Creative Commons licence and your intended use is not permitted by statutory regulation or exceeds the permitted use, you will need to obtain permission directly from the copyright holder. To view a copy of this licence, visit http://creativecommons.org/licenses/by/4.0/.

(C) The Author(s) 2022 